\title{
The motion of Saturn coorbital satellites in the restricted three-body problem
}

\author{
J. Llibre and M. Ollé \\ 1 Dept. Matemàtiques, Universitat Autònoma de Barcelona, Edifici C 08193 Bellaterra, Spain \\ 2 Dept. de Matemàtica Aplicada I, Universitat Politècnica de Catalunya, Diagonal 647, 08028 Barcelona, Spain \\ e-mail: jllibre@mat.uab.es \\ Received 7 March 2001 / Accepted 3 August 2001

\begin{abstract}
This paper provides a description of the motion of Saturn coorbital satellites Janus and Epimetheus by means of horseshoe periodic orbits in the framework of the planar restricted three-body problem for the mass parameter $\mu=3.5 \times 10^{-9}$. The mechanism of existence of such orbits for any value of $\mu>0$ and the Jacobi constant $C$ close to $C\left(L_{3}\right), L_{3}$ being an adequate collinear equilibrium point, is analyzed from two different points of view and a systematic way to compute the horseshoe periodic orbits is also described.
\end{abstract}

Key words. celestial mechanics - planets and satellites - solar system

\section{Introduction}

In 1981 the successful Voyager flights to Saturn confirmed the existence of two small satellites of Saturn, Janus (1908S1) and Epimetheus (1980S3), and provided an estimate of their masses as well as their orbital elements. These coorbital satellites turned out to be librating in horseshoe orbits, in an adequate rotating system. As their semimajor axes are only $50 \mathrm{~km}$ apart, they can approach within $15000 \mathrm{~km}$, but when they are close to each other, their mutual gravitational interaction prevents a collision and switches their orbits. Several authors have dealt with the coorbital motion in the framework of the planar threebody problem: up-to-date numerical values and a detailed history of the discovery is given in Aksnes (1985), Dermott et al. (1980) considered horseshoe orbits to account for the location of narrow rings of Saturn (and other planets), Dermott \& Murray (1981a, 1981b) gave a description of the coorbital motion of 1980S1 and 1980S3 based on a combination of numerical integration and perturbation theory; they studied first the case where the mass of the third body was negligible, and generalized some of the results to include the case where the third body had sufficient mass to affect the other satellite (see also Murray \& Dermott 1999). Later on, Yoder et al. (1983) derived a simple analytic approximation to the motion of all Trojanlike asteroids and applied it to 1980S1 and 1980S3. Rabe (1961) found one horseshoe periodic orbit in the restricted three-body problem for the particular value of the mass parameter $\mu=\mu_{\mathrm{SJ}}$, corresponding to the Sun-Jupiter system, and Taylor (1981) also computed segments of

Send offprint requests to: M. Ollé,

e-mail: olle@ma1.upc.es several families of horseshoe periodic orbits for $\mu_{\mathrm{SJ}}$. On the other hand, other mathematical theories have been developed: Garfinkel $(1977,1978,1980)$ constructed a formal long-periodic solution in the restricted three-body problem, assuming the mass parameter small enough, and such solution embraced in particular horseshaped orbits.

Waldvogel and Spirig studied the problem of Saturn's coorbital satellites by means of a singular-perturbation approach, that is, the motion is initially described by an outer and an inner approximation, valid when the satellites are far apart or close together, respectively; the complete description of the motion requires the matching of both approximations (see Spirig \& Waldvogel 1985; Waldvogel \& Spirig 1988). In a similar way, Petit \& Hénon (1986) dealt with satellite encounters in the framework of Hill's problem from analytical and numerical points of view. Finally, Cors \& Hall (2001) approach this problem analytically by introducing small parameters into the usual three-body equations, truncating higher order terms and deriving dynamical information from the resulting equations.

Our approach deals with the existence of families of horseshoe periodic orbits in the planar circular restricted three-body problem (RTBP). More concretely, the aim of this paper is twofold: (i) first of all, we consider the RTBP where the primaries are Saturn and Janus, that is, with a mass parameter $\mu=3.5 \times 10^{-9}$ (according to Yoder et al. 1989; Nicholson et al. 1992), and Epimetheus (the smaller satellite) is the third body of infinitesimal mass.

We show that the motion of Saturn coorbital satellites is closely related to some periodic orbits of this RTBP problem. We compute new families of stable horseshoe periodic orbits which approach the actual 
motion of $1980 \mathrm{~S} 1$ and $1980 \mathrm{~S} 3$; so the coorbital motion is already reflected in this simple model. In fact, Dermott \& Murray (1981a, 1981b) did so in some way, and we will compare our results with theirs. However, in this paper, we present a mechanism of generation of horseshoe periodic orbits as a transition from the $\mu=0$ case to the $\mu>0$ and small one. This mechanism gives answer to the natural question about the origin and location of these horseshoe periodic orbits. We carry out a numerical exploration for different values of $\mu$, we will compute some families of horseshoe periodic orbits in a systematic way, and we will describe some properties of their behaviour. This exploration reveals that the set of families of horseshoe periodic orbits has a rich structure as well as the appearance of bifurcation phenomena. More concretely, according to their stability, we will show that many bifurcations appear which are very close to each other. These features were not considered by Dermott and Murray.

(ii) We are also concerned with the existence of families of horseshoe periodic orbits for other values of the mass parameter in the framework of the restricted problem. So, we will discuss the existence of such orbits for any value of the mass parameter $\mu$ varying from 0 to $1 / 2$. As this question is directly related to the variation of the Jacobi constant, we will also describe the evolution of the families of horseshoe periodic orbits for a fixed value of the mass parameter and varying the Jacobi constant $C \in\left[3, C_{1}\right)$.

In particular, we discuss the relation between the horseshoe periodic orbits and the invariant manifolds of the Lyapunov orbits born from the collinear points (specially from $L_{3}$ ). We recall that for each collinear equilibrium point $L_{i}, i=1,2,3$, the infinitesimal oscillations around $L_{i}$ are continued along a family of plane retrograde periodic orbits surrounding $L_{i}$, the so called family of Lyapunov periodic orbits (see Szebehely 1967).

The paper is organized as follows: the mechanism of generation of horsehoe periodic orbits, for small $\mu>0$, is described in Sect. 2. The numerical exploration for the motion of the coorbital satellites Janus and Epimetheus (considering the circular RTBP as a simplified model) as well as the properties of horseshoe periodic orbits (for $\mu>$ 0 and very small) are carried out in Sect. 3. In Sect. 4 , we discuss the horseshoe periodic orbits for any value of $\mu$, and finally, we draw some conclusions in Sect. 5 .

\section{Origin and location of horseshoe periodic orbits. From $\mu=0$ to $\mu>0$ small}

\subsection{The restricted three-body problem}

We consider a system of three bodies in an inertial (also called sidereal) reference system: two bodies (called primaries) of masses $1-\mu$ and $\mu$ (in suitable units), describing circular orbits about their common center of mass (the origin of coordinates) in a plane, and a particle of infinitesimal mass which moves in the same plane under the gravitational effect of the primaries but has negligible effect on their motion. The problem of describing the

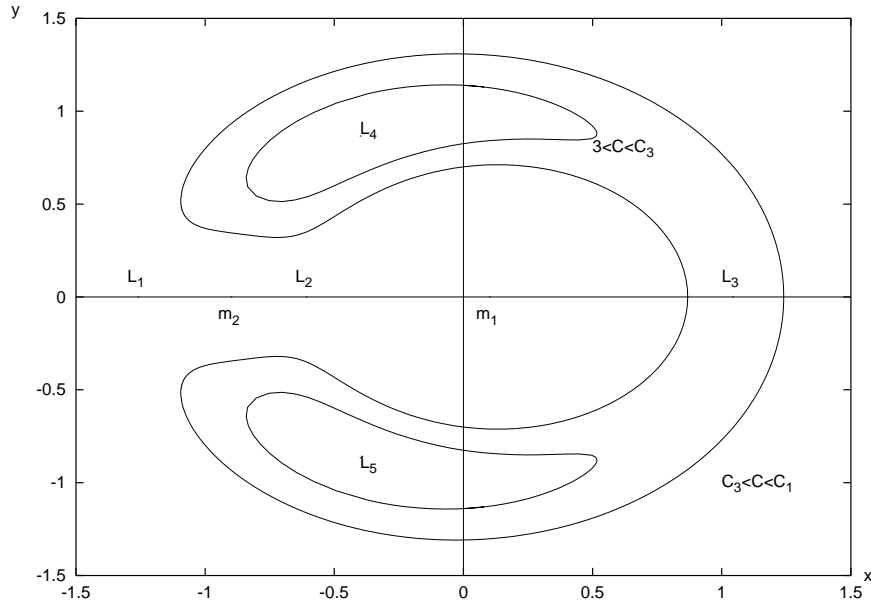

Fig. 1. Zero velocity curves for $\mu>0$. The motion is possible outside the region enclosed by the $z v c$.

motion of the particle is the planar circular restricted three-body problem (RTBP). The equations of motion in a rotating (also called synodical) system of coordinates, $x$ and $y$, which rotates with the primaries are, in suitable units (see Szebehely 1967)

$x^{\prime \prime}-2 y^{\prime}=\frac{\partial \Omega}{\partial x}$

$y^{\prime \prime}+2 x^{\prime}=\frac{\partial \Omega}{\partial y}$

where

$\Omega(x, y)=\frac{1}{2}\left(x^{2}+y^{2}\right)+\frac{1-\mu}{r_{1}}+\frac{\mu}{r_{2}}+\frac{1}{2} \mu(1-\mu)$,

and $r_{1}^{2}=(x-\mu)^{2}+y^{2}, r_{2}^{2}=(x-\mu+1)^{2}+y^{2}$ are the distances between the particle and the bigger and smaller primaries respectively, and / stands for $\mathrm{d} / \mathrm{d} t$. It is well known that these equations have the so called Jacobi first integral

$x^{\prime 2}+y^{\prime 2}=2 \Omega(x, y)-C$,

and 5 equilibrium points: the collinear points, $L_{1}, L_{2}$ and $L_{3}$, and the equilateral ones, $L_{4}$ and $L_{5}$. If one computes the value of the Jacobi constant at the equilibrium points $C_{i}=C\left(L_{i}\right)$ for any value of $\mu \in(0,1 / 2)$, one has

$3=C_{4}=C_{5}<C_{3}<C_{1}<C_{2}$,

and $C_{3}=C_{1}$ for $\mu=1 / 2$.

Throughout this paper, a horseshoe periodic orbit will be a periodic solution in which the particle follows a path which surrounds only the equilibrium points $L_{3}, L_{4}$ and $L_{5}$ and has only two crossings with the $x$ axis, which turn out to be orthogonal; that is, for $t=0$ and $t=T / 2$, $\mathrm{T}$ being the period, the orbit has $\left(x_{i}, 0\right)$ as position and $\left(0, y_{i}^{\prime}\right)$ as velocity, for certain $x_{i}$, and $y_{i}^{\prime}, i=1,2$. It is well known that the equations of motion of the RTBP satisfy the symmetry

$\left(x, y, x^{\prime}, y^{\prime}, t\right) \rightarrow\left(x,-y,-x^{\prime}, y^{\prime},-t\right)$ 


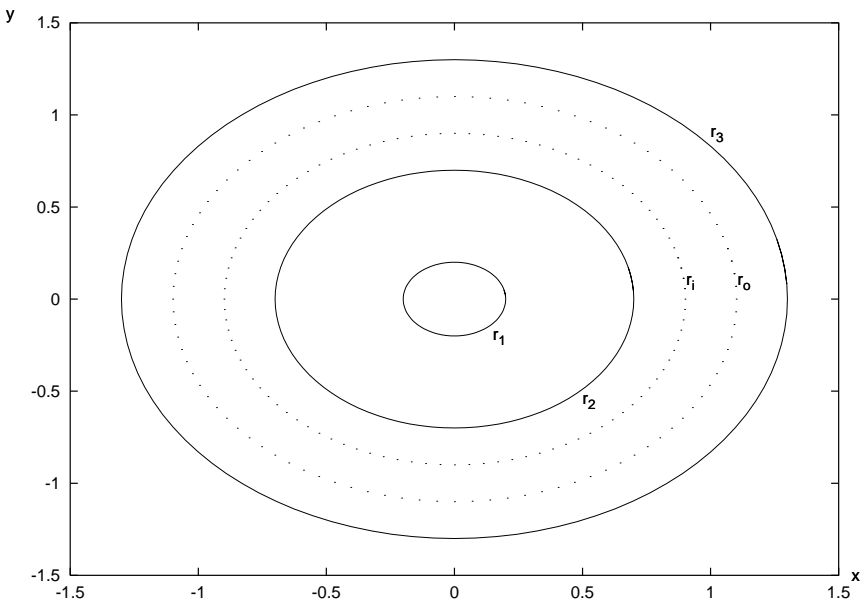

Fig. 2. For $\mu=0$ and $C>3$, the motion is possible inside the disk of radius $r_{\mathrm{i}}<1$ and outside the disk of radius $r_{\mathrm{o}}>1$. We also plot three circular periodic orbits of radius $r_{1}$ (retrograde), $r_{2}$ (direct) and $r_{3}$ (retrograde).

and that any solution with two orthogonal crossings with the $x$ axis (at $t=0$ and $t=T / 2$ ) becomes symmetric with respect to the $x$ axis, and periodic of period $T$. So, from now on, any horseshoe periodic orbit will be considered for $t \in[0, T / 2]$.

On the one hand, we want to know the suitable region in the plane $(x, y)$ to look for horseshoe periodic orbits. To do so, we just recall that for any value of $\mu$, one has the possible regions of motion (whose boundaries are called zero velocity curves $-z v c-$ ) according to the value of the Jacobi constant $C$ (see more details in Szebehely 1967, Chapter 4). An examination of those possible regions of motion shows that horseshoe periodic orbits may take place only for $C<C_{1}$ and for values of $x$ and $y$ close to the zero velocity curves (we will specify the meaning of close later on). At this point, we remark that the natural range of $C$, for the horseshoe periodic orbits, is $3<C<C_{1}$ (see Fig. 1), which was the actual range taken in Dermott \& Murray (1981a, 1981b), but we will also prove that there exist horseshoe periodic orbits for $C \leq 3$.

On the other hand, for a fixed $C<C_{1}$ we may expect to have many horseshoe periodic orbits for $\mu>0$ and very small. The reason remains in the RTBP for $\mu=0$, as we shall see in the next subsection.

We briefly recall the RTBP, with the particular value of the mass parameter $\mu=0$. In this case, any point with $x^{\prime}=y^{\prime}=0$ on the circle $S^{1}$ (centered at the origin and of radius one) is an equilibrium point. The possible regions of motion are: outside the ring of radius $r_{\mathrm{i}}<1$ and $r_{\mathrm{o}}>1$ which are solutions of the equation $r^{3}-C r+2=0$, with $r>0$, for $C>3$; the plane without crossing the circle $S^{1}$, for $C=3$, and the whole plane for $C<3$ (see Fig. 2).

\subsection{Mechanism of generation of horseshoe periodic orbits}

It is well known (see Szebehely 1967) that, for $\mu=0$, any periodic orbit of the RTBP in synodical coordinates comes

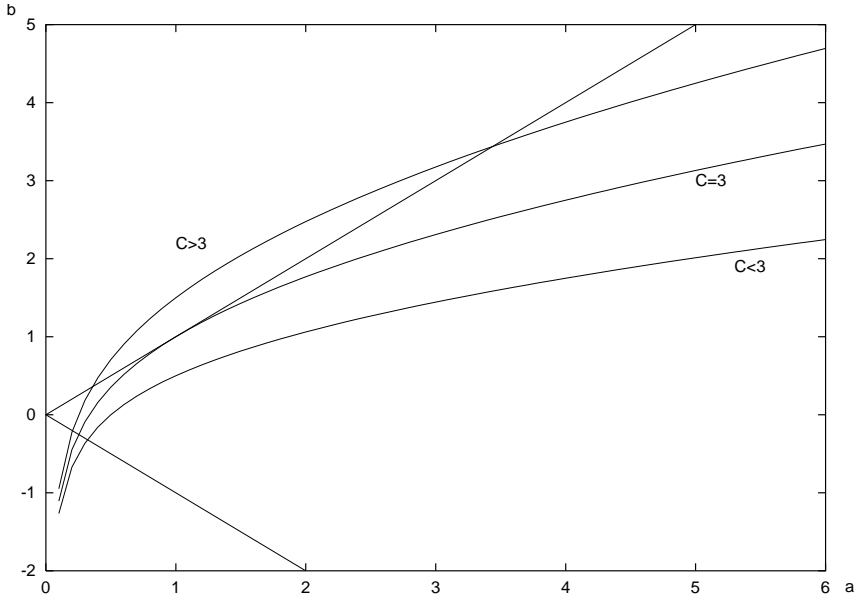

Fig. 3. For a fixed $C$, any value of $a=(p / q)^{2 / 3}$, with $p, q \in N$ and $b$ in the corresponding curve are the semiaxes of a sidereal ellipse which becomes a periodic orbit in synodical coordinates.

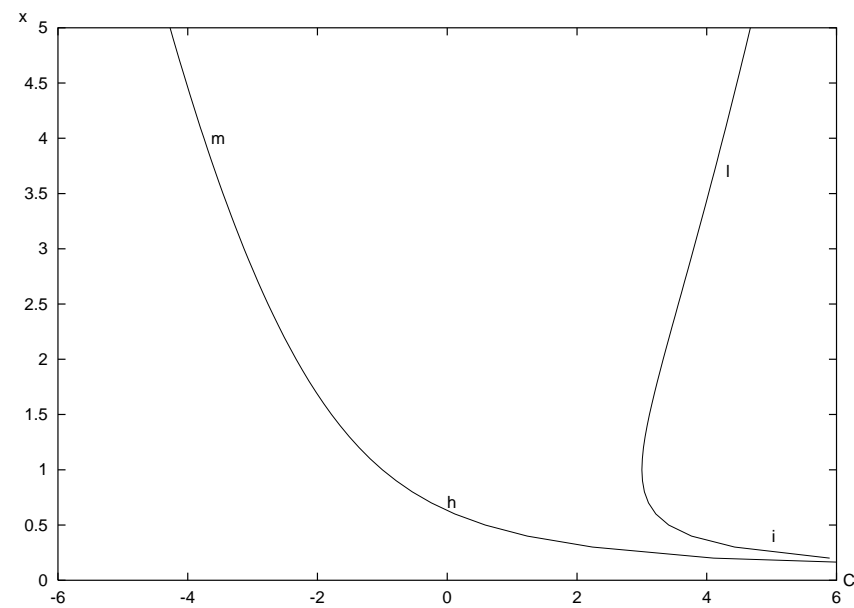

Fig. 4. Characteristic curves of families $l, i, h$ and $m$ of synodical circular orbits.

from rotating a circular orbit or a suitable elliptical one in the sidereal (inertial) frame. More concretely, for a fixed value of $C$, only the sidereal ellipses (that is, in sidereal cordinates), with semimajor axis $a$, semiminor axis $b$ and eccentricity $e$ given by the relation

$\pm b= \pm a \sqrt{1-e^{2}}=\frac{C \sqrt{a}}{2}-\frac{1}{2 \sqrt{a}}$

(see Fig. 3), with $a=(p / q)^{2 / 3}$, for some $p, q \in N$ (that is with rational mean motion), give rise to periodic orbits in synodical coordinates; of course, $a$ and $b$ are always positive but the signs,+- assign a sense to describe the motion: $(+)$, the corresponding sidereal orbit is direct, and $(-)$, the sidereal orbit is retrograde.

In particular, when $e=0$, a circular orbit in sidereal coordinates also becomes circular in synodical ones; and the characteristic curves (that is a curve whose points provide initial conditions of periodic orbits) of the circular synodical symmetric periodic orbits are given by

$C=\frac{1}{a}+2 \sqrt{a}(l-i)$, 

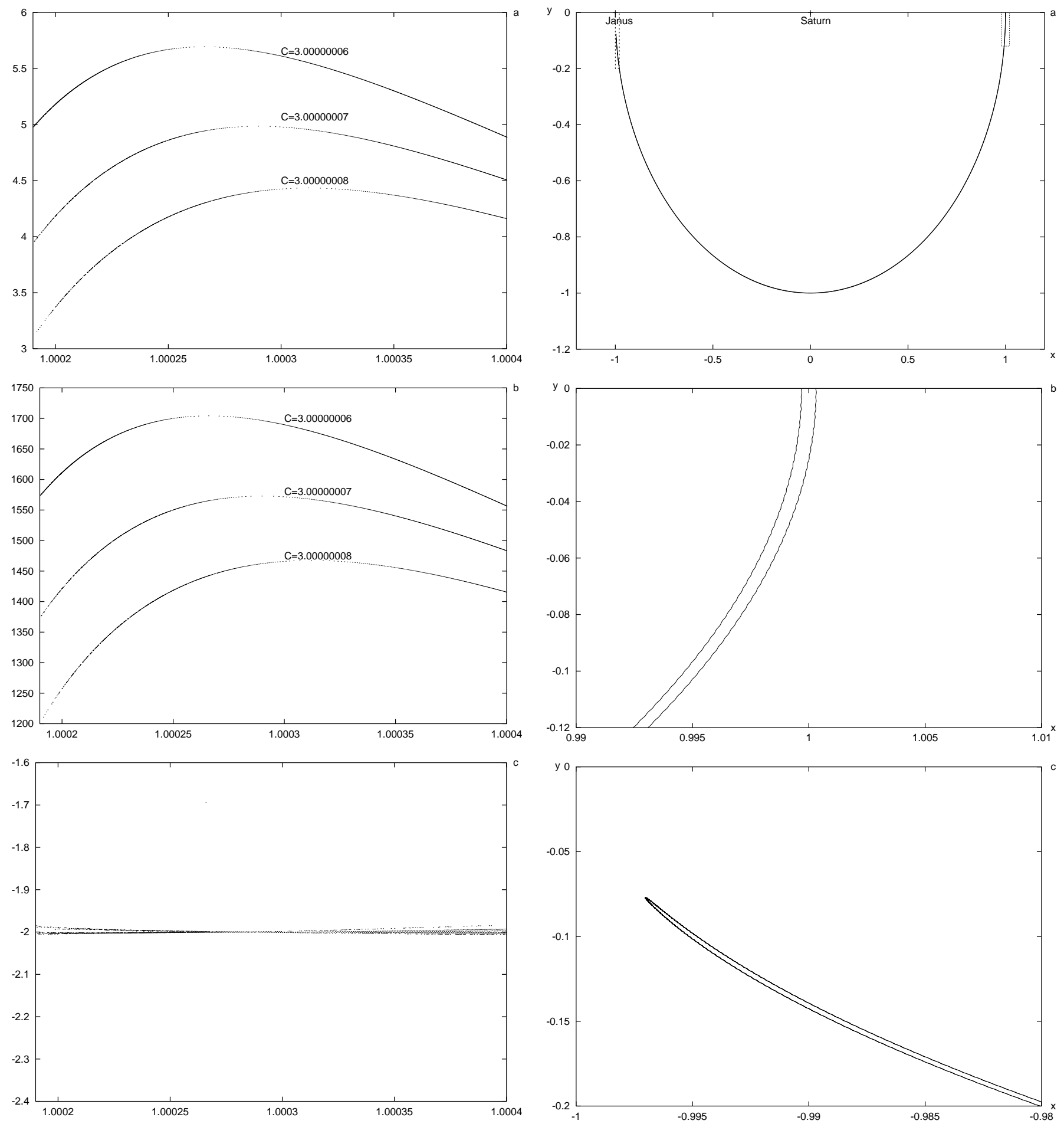

Fig. 5. Initial conditions $x$ versus the minimum angular separation $\theta_{\min }$ of horseshoe periodic orbits for different values of $C \in\left(C_{3}, C_{1}\right)$ a) For every horseshoe periodic orbit computed, we plot the value of the half period $T / 2$, in days, b) and the stability parameter $\mathbf{c}$ ).

$C=\frac{1}{a}-2 \sqrt{a}(m-h)$,

(see Fig. 4). Thus, we recover the well known families:

$l$ periodic orbits around $m_{1}$ and $m_{2}$, its motion is retrograde in the synodical system and direct in the sidereal system;

Fig. 6. a) A horseshoe periodic orbit suitable for Janus and Epimetheus. b) Detail. c) The effect of return when both satellites approach each other.

$i$ direct periodic orbits around $m_{1}$ in both systems, synodical and sidereal;

$h$ retrograde periodic orbits around $m_{1}$ in both systems;

$m$ retrograde periodic orbits around $m_{1}$ and $m_{2}$ in both systems. 


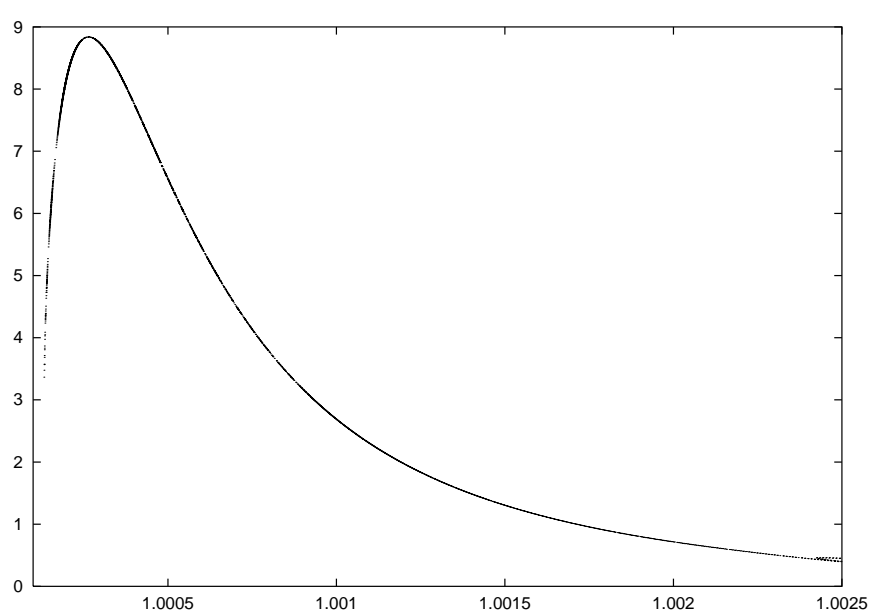

Fig. 7. Family of horseshoe periodic orbits for $\mu=6.5 \times 10^{-9}$ and $C=3.000000065$; initial condition $x$ versus $\theta_{\min }$.

As far as synodical circular orbits are concerned, Fig. 4 shows that for a fixed value of the Jacobi constant $C>3$, we have three circular orbits of increasing radius $r_{1}$ (retrograde), $r_{2}$ (direct) and $r_{3}$ (retrograde) in families $h, i$ and $l$, respectively (see these orbits in Fig. 2). Now we pay attention to the circular orbits with radius $r_{2}$ and $r_{3}$, for $C>3$ and $C$ close to 3 ; we expect that for $\mu>0$ and very small, we may have a horseshoe periodic orbit with the two perpendicular crossings $x_{0}=x(t=0)>0$ and $x_{1}=x(t=T / 2)>0$ very close to the values $r_{2}$ and $r_{3}$. According to the singular perturbation theory carried out by Spirig and Waldvogel, these two circular orbits, outside a neighborhood of the small primary, would become the outer approximation of the horseshoe periodic orbit. It is also clear that the effect of the small primary with mass $\mu>0$ may cause the typical shape of "return" close to the primary, originating in the RTBP that the infinitesimal mass passes from a nearly circular orbit of radius $r_{2}$ to a nearly circular orbit of radius $r_{3}$ and vice versa in consecutive approaches to the small primary $m_{2}$. As we shall see in the next section, this is precisely the case. So, we may say that the two circular orbits, of radius $r_{2}<1<r_{3}$, and $r_{2}, r_{3}$ close to 1 , generate one horseshoe periodic orbit.

The same reasoning applies to synodical orbits obtained from rotating sidereal ellipses. However, as Fig. 3 shows, they exist for any value of $C$, so for a fixed value of $C$ close to 3 (greater than, equal to, or smaller than 3 ), we have a continuous set of synodical orbits; so it is likely that many pairs of them are the natural candidates to provide (outside a neighborhood of the small primary) horseshoe periodic orbits for $\mu>0$ and small. In summary, we have given a natural mechanism to generate horseshoe periodic orbits, and we can expect for a fixed value of $\mathrm{C}$ close to 3 , and $\mu>0$ small, many horseshoe periodic orbits. We will obtain them in the next section.

\section{Computation of horseshoe periodic orbits for $\mu>0$ and very small}

\subsection{Orbital motion of Janus and Epimetheus}

Our aim in this section is to compute stable horseshoe periodic orbits that somewhat fit the real orbit described by 1980 S 1 and 1980 S3.

We will take into account that 1980S3 describes a horseshoe periodic orbit of period 2928.6 days, the minimum angular separation between both satellites is 5.6 degrees, and both satellites describe elliptical orbits of semiaxis $151.472 \mathrm{~km}$ and $151.422 \mathrm{~km}$ and eccentricity 0.007 and 0.009 for 1980S1 and 1980S3 respectively (see Murray \& Dermott 1999).

So, in our simplified model, we consider the RTBP with Janus (1980S1) as the small primary, with mass $\mu=3.5 \times 10^{-9}$, Saturn as the big one with mass $1-\mu$, both describing circular orbits around their common center of mass, and the coorbital satellite Epimetheus as the infinitesimal body. For this value of $\mu$ we have that the $x$ coordinate of the collinear singular points and their Jacobi constant values are

$$
\begin{aligned}
& x\left(L_{2}\right)=-.99894763, \quad C_{2}=3.000009966, \\
& x\left(L_{1}\right)=-1.00105309, \quad C_{1}=3.000009961, \\
& x\left(L_{3}\right)=1.0000000015, C_{3}=3.000000007 .
\end{aligned}
$$

In order to compute horseshoe periodic orbits, we fix a value of the Jacobi constant $C$ and we obtain them. We remark that we have not computed families of horseshoe periodic orbits when varying $C$, since as we shall see later, the initial conditions of horseshoe periodic orbits (for fixed $C$ ) are too close to each other.

So we fix a value of $C$. If $C_{3}<C<C_{1}$ then we determine the values $x_{\mathrm{i}}>0$ and $x_{\mathrm{o}}>0$ of the intersection between the zero velocity curve and the $x$ axis. We start with a value of $x$ very close to and bigger than $x_{\mathrm{o}}$; from (3) we have the corresponding value of $y^{\prime}$ since we start at an orthogonal crossing, that is, the orbit begins at $\left(x, 0,0,-y^{\prime}\right)$. Then, we integrate the equations of motion until the orbit again crosses the $x$ axis, at a point $\left(\bar{x}, 0, \bar{x}^{\prime}, \bar{y}^{\prime}\right)$. Afterwards, we increase the value of $x$ by an increment of $10^{-7}$ and we go on until a change in sign of $\bar{x}^{\prime}$ is found. We just compute then the value of $x$ (between the last two values of $x$ ) of the initial condition for the symmetric periodic orbit. Of course, we can go on increasing the value of initial $x$ and in this way we obtain all the horseshoe periodic orbits in a given interval of $x$ (where the separation between two consecutive values of initial conditions $x$ is at least $10^{-7}$ ). As pointed out by Dermott \& Murray (1981a), for different values of initial $x$, the infinitesimal body can enter a neighborhood of the small primary of it can even collide with it. We have also detected orbits which enter a neighborhood of the big primary or the particle even collides with it; thus we have regularized the differential equations of motion using LeviCivitta variables in order to regularize a neighborhood of either primary (see Szebehely 1967) whenever necessary. 

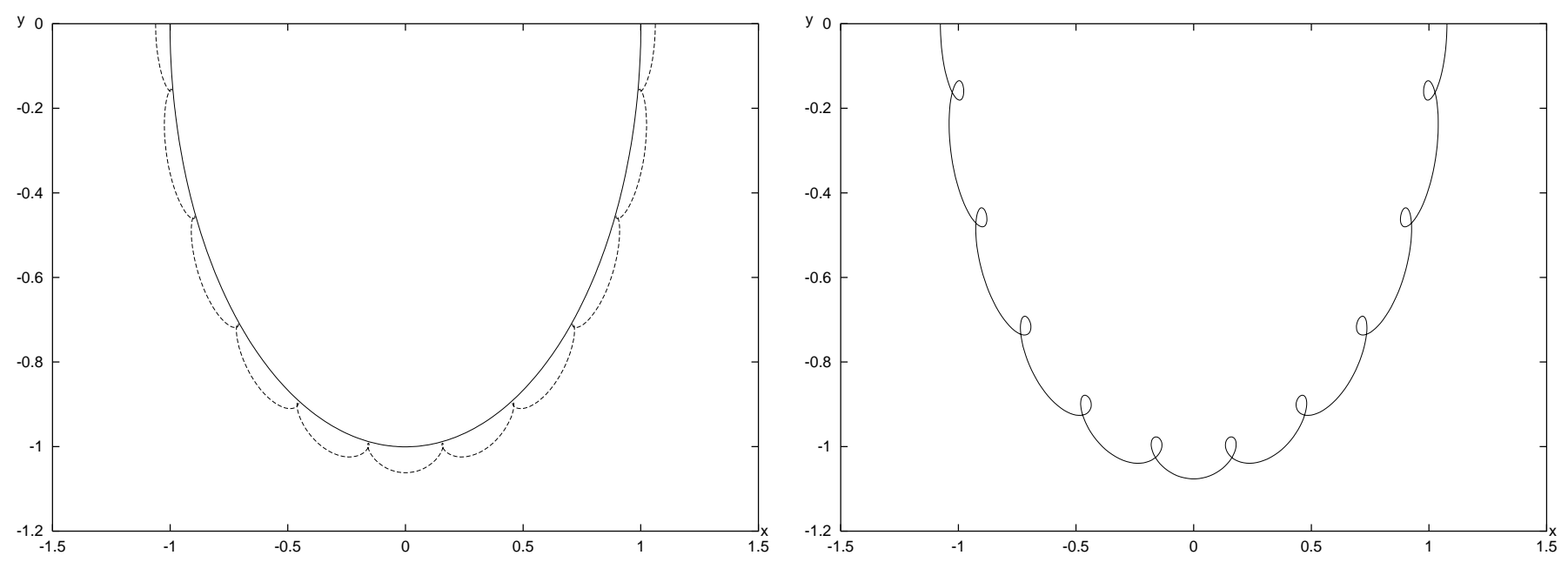

Fig. 8. Synodical periodic orbit obtained from rotating a sidereal ellipse with $a=(p / q)^{2 / 3}, q=20, p=21$, for $\mu=0$. We plot the orbit for half period, and the ellipse is described $10=q / 2$ times. a) For $C=3.000001$, there is also the $z v c$ (circular curve); the close points to the $z v c$ correspond to the pericenter of the ellipse and the further ones to the apocenter. b) For $C=2.999$, there is no $z v c$.
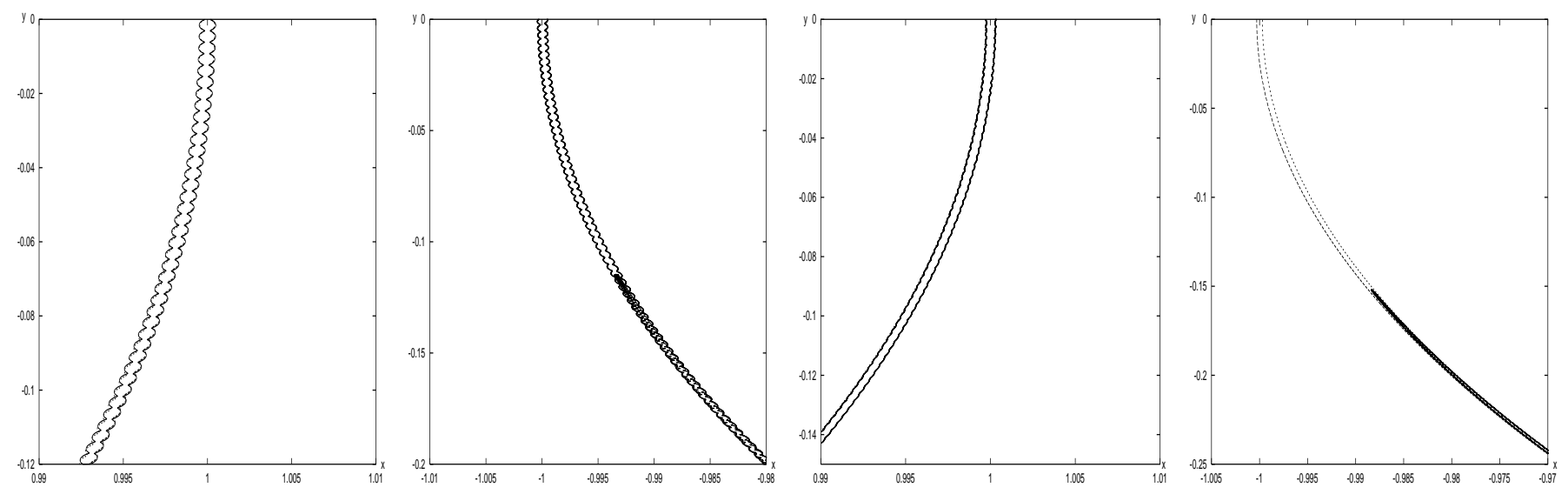

Fig. 9. A piece of a horseshoe periodic orbit (continuous line) for $\mu=6.5 \times 10^{-9}$ and the approximating elliptical orbits a) and circular orbits c) for $\mu=0$ (dotted lines). Another piece, with the return point, close to the small primary $\mathbf{b}$ ) and $\mathbf{d}$ ).

As we are interested in looking for stable horseshoe periodic orbits which fit the motion of the coorbitals, once the periodic orbit is obtained, we have determined its linear stability. In order to do so, we integrate the variational equations, we compute the monodromy matrix $M$ and we recall that the stability parameter is defined by $\alpha=2-\operatorname{tr} M$. It is well known that the orbit is linearly stable if $\alpha$ satisfies $-2<\alpha<2$ and unstable, otherwise (see for instance Bray \& Goudas 1967, where it is shown how the computation of $\alpha$ through the monodromy matrix for half period is enough).

In a similar way, if $3 \leq C<C_{3}$, the $z v c$ does not cross the $x$ axis, and if $C<3$, then there is no $z v c$ at all. In both cases, we consider as the starting $x$ a value very close to and bigger than $x\left(L_{3}\right)$.

We describe now the results. We have computed horseshoe periodic orbits for different values of $C$ ranging from values less than 3 to $C_{1}$. We show, for instance, in Fig. 5 the initial value $x$ for the families of horseshoe periodic orbits computed for different values of $C \in\left(C_{3}, C_{1}\right)$.
There exist thousands of horseshoe periodic orbits but as they are so close to each other and for sake of clarity, we only show a particular range which includes the desired value of $r_{1}=1.00033$ (which corresponds to the orbital element of Epimetheus in rotating coordinates). As we can see, for $x \in[1.0002,1.0004]$ there are many horseshoe periodic orbits with suitable values of the half-period (close to 1464), minimum angular separation (close to 5.6 degrees) although there are few which are stable. We plot in Fig. 6a selected stable horseshoe periodic orbit with $x(t=0)=1.0002979809, x(T / 2)=0.9996731232$, $T=1465.3$ days, and for which the minimum angular separation is 4.42 degrees; we also see the effect of "return" when 1980S3 approaches 1980S1 (see Fig. 6c). The eccentricity for the orbit selected is $0.9 \times 10^{-4}$ and we remark that we have considered here a simple model where the big satellite describes an "inertial circular orbit". Of course the orbits of the moons considered are both slightly eccentric, so this means that a better model should take into account this effect (see Sect. 5). However, since our goal in 

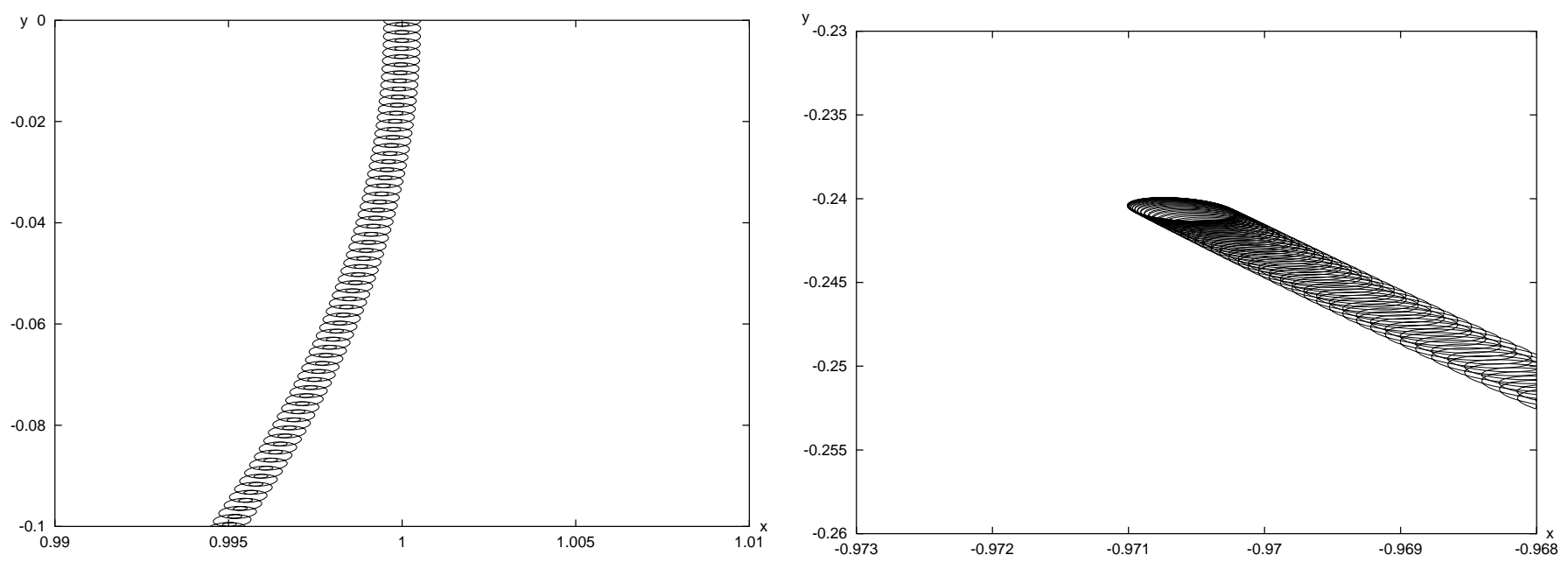

Fig. 10. Two pieces of a horseshoe periodic orbit for $\mu=6.5 \times 10^{-9}$ and $C=2.9999999$.

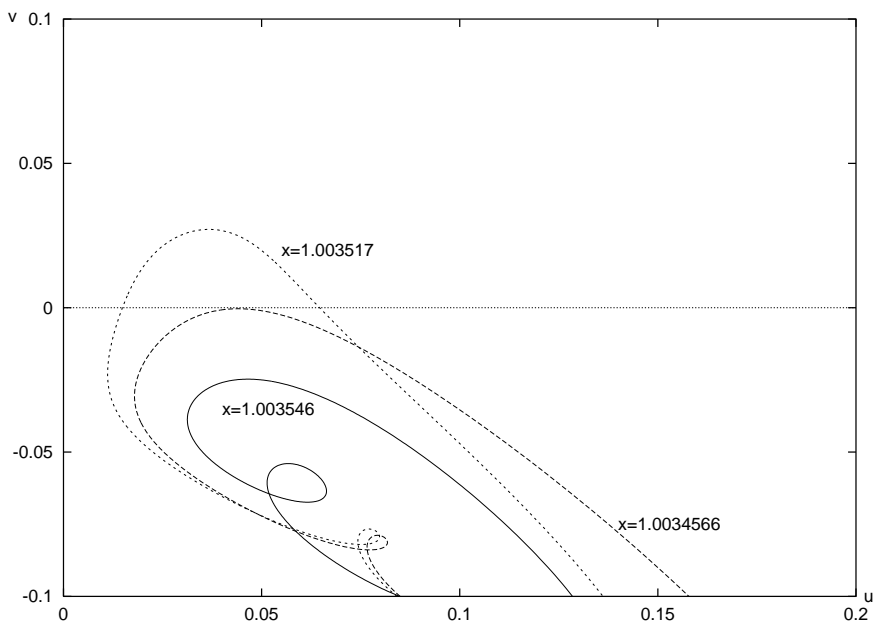

Fig. 11. $\mu=6.5 \times 10^{-9}$ and $C=3.000000065$. We use LeviCivitta coordinates $u, v\left(m_{2}\right.$ is located at the origin); the return point oscillates in the $y<0$ region and in the $y \geq 0$ one.

this paper is to analyze the existence of this kind of motion inside the circular RTBP, we can conclude that, inside the circular RTBP, there exist stable horseshoe periodic orbits that describe qualitatively the coorbitals' motion.

\subsection{Horseshoe periodic orbits as periodic orbits of the RTBP}

In this section the horseshoe periodic orbits are regarded as particular periodic orbits of the RTBP for $\mu>0$ and very small. We want to check the mechanism of generation of horseshoe periodic orbits described in Sect. 2 and analyse their properties when considering families of them. Since the same behaviour is obtained for different values of $\mu>0$, we fix, for example, $\mu=6.5 \times 10^{-9}$; in this case, $C_{3}=3.000000013, C_{1}=3.00001504$, and we plot the family of horseshoeperiodic orbits obtained for
$C=3.000000065 \in\left(C_{3}, C_{1}\right)$ in Fig. 7. In this context, four remarks must be made:

1. The typical shape of horseshoe periodic orbits will depend on two features according to the value of $C$ : the existence of $z v c$ and the outer approximation. In particular, for $\mu=0$, a sidereal circle turns out to be a circular synodical orbit and it is well known that a sidereal ellipse with semimajor axis $a=(p / q)^{2 / 3}$ gives rise to a periodic orbit in synodical coordinates with synodical period $T=2 \pi p$; and during this time, the sidereal ellipse is traversed $q$ times. We show a simple example with $\mu=0$ and $a=(p / q)^{2 / 3}, q=20, p=21$ in Fig. 8 .

For $\mu>0$ and small, we expect the same shape for the horseshoe periodic orbits (outer approximation). We consider first the case $C_{3} \leq C<C_{1}$. The zvc restricts the region of motion and the outer approximation of the horseshoe periodic orbits will be either rotating ellipses or circles. We show the typical shape of a horseshoe periodic orbit with $x(t=0)=1.000158887628$ and $x(T / 2)=0.999841160777$ in Fig. 9 (a and b); we have computed the semiaxis $a$ and $b$ from (4) for the generating ellipses (for $\mu=0$ ) that give the initial conditions $x=1.0002043120$ and $x=0.9997984943$ and we plot them as well in Fig. 9 ( $\mathrm{a}$ and b). We also show in Fig. 9 (c and $\mathrm{d}$ ) the horseshoe periodic orbit with initial conditions (1.000294264596,0,0,-0.0004557622) such that $x(T / 2)=0.999795834471204, y^{\prime}(T / 2)=0.00045569264$; we also plot the two generating circles, that is, two circular orbits given by the two values of $r$ of Eq. (6) for $\mu=0$ and the same value of $C$, that is, $x=r=1.00029444$, $y^{\prime}=-0.000441626$ (as a retrograde circular orbit) and $x=r=0.999705659, y^{\prime}=0.0008486589$ (as a direct circular one). We can see that both approximations agree quite well with their corresponding horseshoe periodic orbits when both satellites are far apart from each other.

We fix now $C$ such that $3<C<C_{3}$. There is still the zero velocity curve which does not cross the $x$ axis anymore; there are still the approximate rotating ellipses or circles (from $\mu=0$ ). 

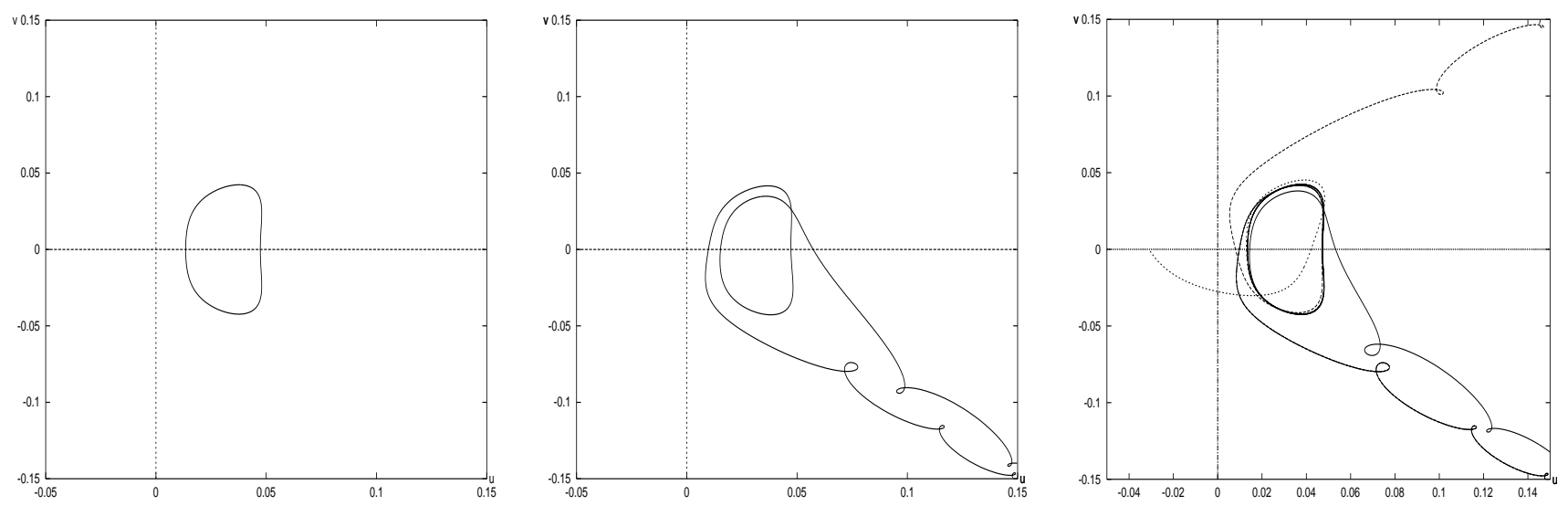

Fig. 12. $\mu=6.5 \times 10^{-9}$ and $C=3.000000065$ and Levi-Civitta coordinates $u, v$. a) Lyapunov orbit of $L_{2}$ for this value of $C$. b) Orbit with $x(t=0)=1.0035181$. c) The orbit with $x(t=0)=1.0035181044137$ turns down in a horseshoe shape, the one with $x(t=0)=1.0035181044138$ turns upwards and the one with $x(t=0)=1.00351810441385$ is close to a collision with the small primary.
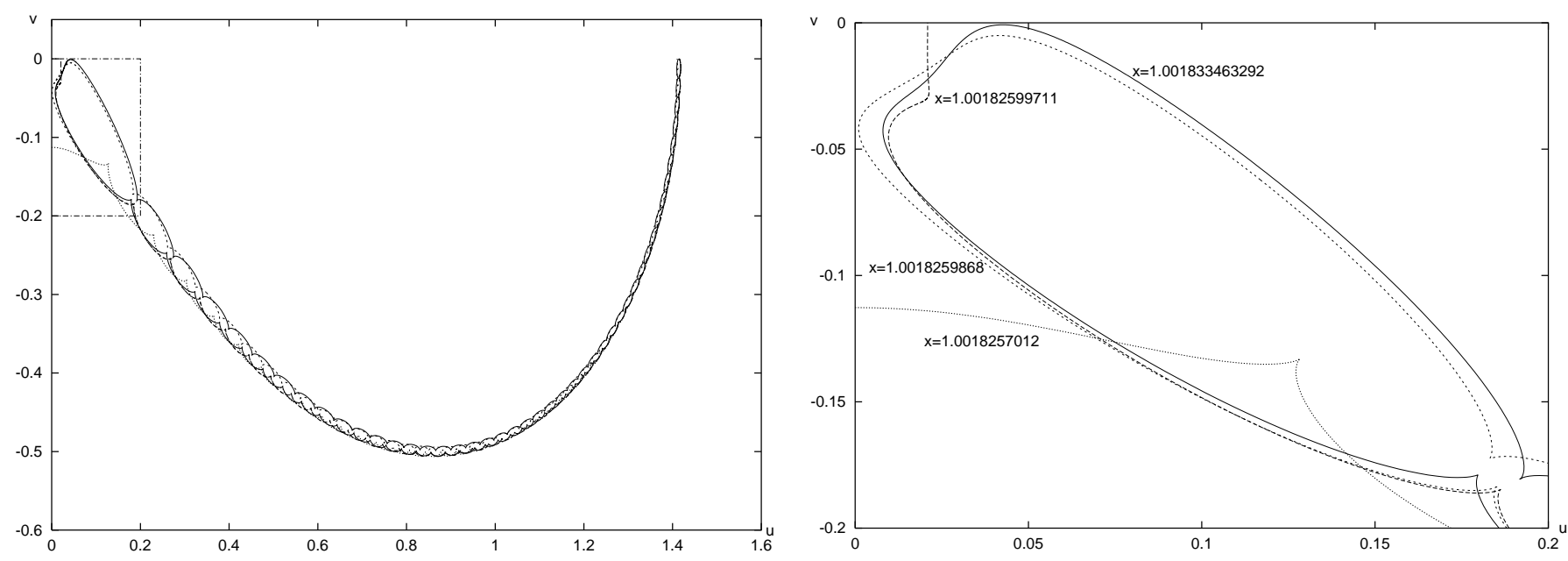

Fig. 13. a) Horseshoe periodic orbits close to the Lyapunov orbits of $L_{1}$ and $L_{2}$ for $\mu=6.5 \times 10^{-9}$ and $C=3.00001$. b) Detail.

Finally for $C \leq 3$, there is no $z v c$ at all and the horseshoe periodic orbits may be approximated only by rotating ellipses (from $\mu=0$ ). We show a typical horseshoe periodic orbit in Fig. 10; the loops (the projections on the $x-y$ plane) intersect each other since there is no restriction from the $z v c$.

2. According to their stability, the obtained horseshoe periodic orbits for $C<3$ are unstable. As $C$ is increased, some stable orbits appear and, in general, many horseshoe periodic orbits are very close to critical ones in the sense that their stability parameter satisfies $\alpha=-2$; therefore there may appear many bifurcations which we have not followed since their initial conditions are too closely located.

3. For a fixed value of $C$, the evolution of horseshoe periodic orbits when increasing $x$ is the following:

(i) For $C_{3}<C<C_{1}$, the $y<0$ coordinate of the return point of each horseshoe periodic orbit increases up to 0 , that is $\theta_{\min }$ decreases to 0 (see Fig. 7), which means that the infinitesimal body becomes closer to the small primary.
We describe now with more detail how this $y$ coordinate of the return point goes to zero and how the horseshoe periodic orbits evolve. As the particle passes close to the small primary, $m_{2}$, we use the Levi-Civitta coordinates $u, v, \dot{u}, \dot{v}$ that regularize the collision with $m_{2}$; we note that $u$ and $v$ are the space cordinates and $\dot{u}$ and $\dot{v}$ describe the velocity with respect the new independent time variable (see Szebehely 1967). We fix $C=3.000000065 \in\left[C_{3}, C_{1}\right]$; for $x \in[1.000131789,1.003456]$ (the left $x$ of the interval corresponding to the first horseshoe periodic orbit computed), there are many horseshoe periodic orbits, so both crossings with the $x$ axis are orthogonal and take place with $x>0$. However, as $x$ increases, the $y$ coordinate of the return point is closer to 0 . The influence of $m_{2}$ is most important now. As the initial condition $x$ increases further on, the corresponding orbit (not necessarily periodic) described by the particle looks like a horseshoe orbit. However, for $x=1.0034566$, the particle has a return point which is tangent to the $x$ axis with $x=-0.9986$, that is, the return point takes place for $y=0, x<0$ on the right of $m_{2}$ and very close to it. For $x \in[1.00345665,1.003518]$, the return point 

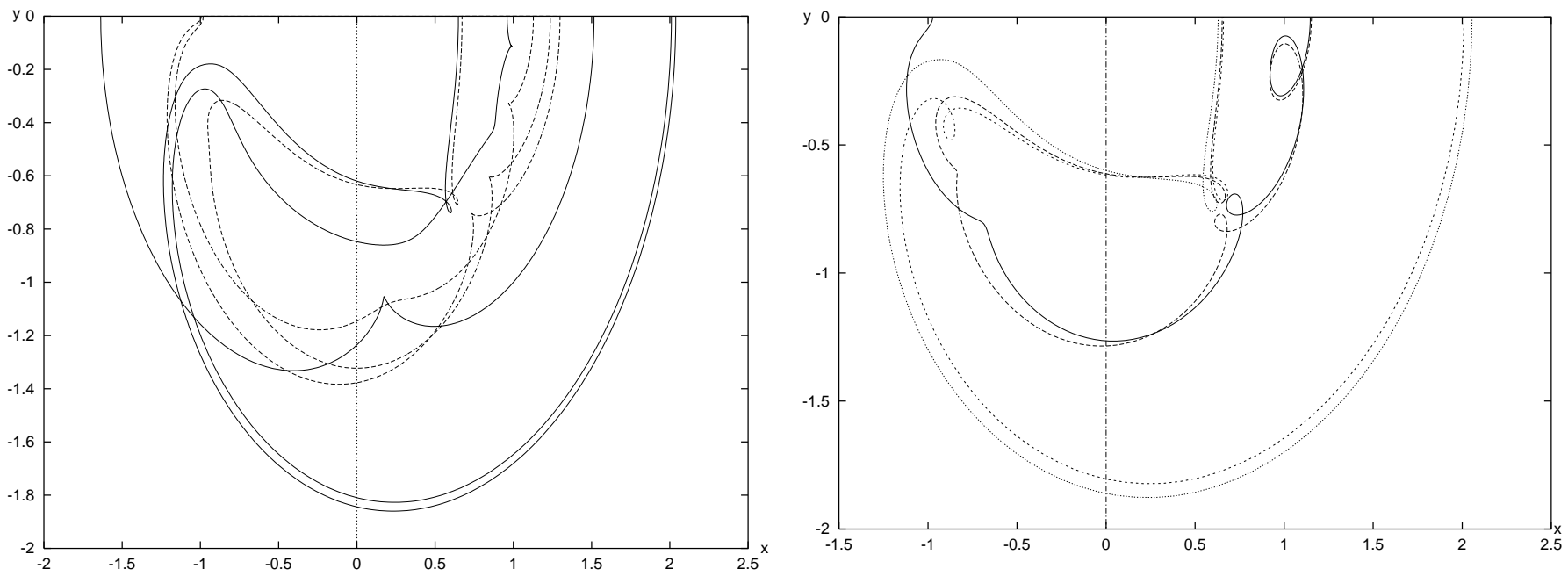

Fig. 14. Evolution of the corresponding periodic orbits when increasing $x$, for $\mu=0.01$ and a) $C=3.0199 \in\left(C_{3}, C_{1}\right)$; and b) $C=3.01<C_{3}$.
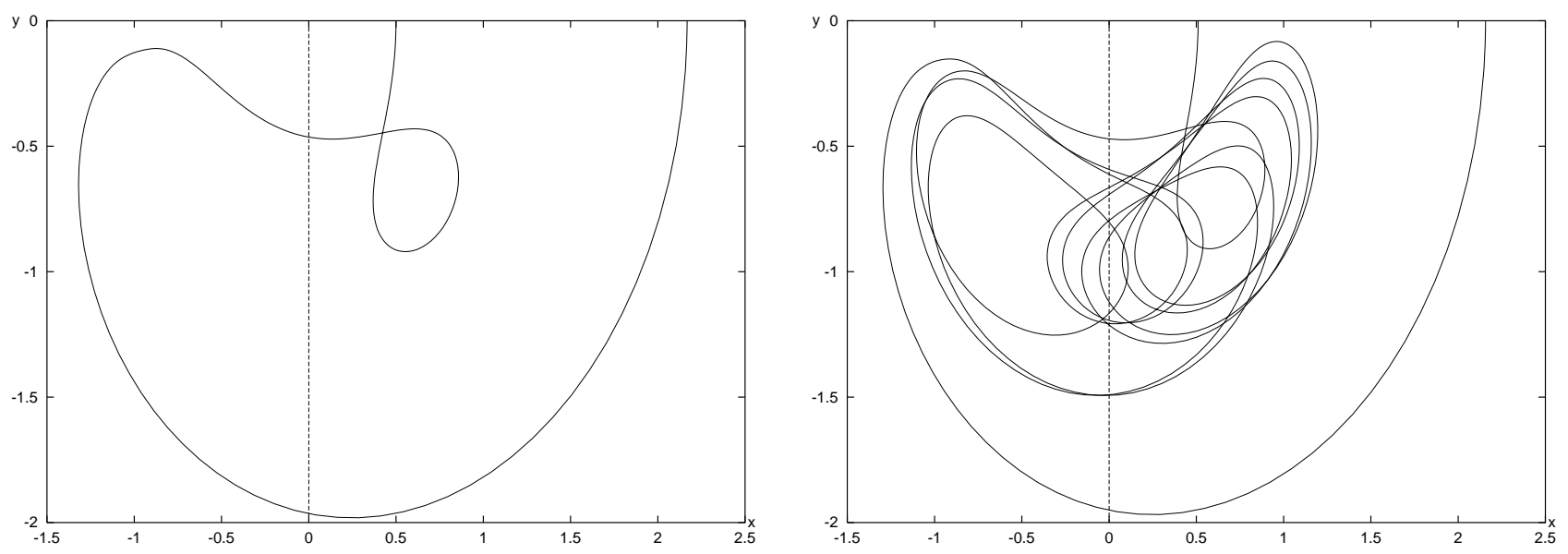

Fig. 15. Horseshoe periodic orbit for $\mu=0.01$ and $C=2.9$. a) $x=2.16792965275$. b) $x=2.1571843149$.

oscillates and takes place close to $m_{2}$ but either in the $y \geq 0$ region or in the $y<0$ one (see Fig. 11). A careful exploration (using quadruple precision) shows that the Lyapunov orbit around $L_{2}(x(t=0)=-0.997761039$, $x(T / 2)=-0.99981859$, see Fig. 12a) also has its effect on the dynamics in this region and a very thin range of variation in the $x$ variable gives rise to different kinds of orbits. More concretely, for $x=1.0035181$ the corresponding orbit has a horseshoe shape but surrounds the Lyapunov orbit of $L_{2}$ once (see Fig. 12b); as $x$ increases a little bit, the corresponding orbit for $x=x_{1}=1.0035181044137$ surrounds the Lyapunov orbit twice, and turns downwards in a horseshoe shape (see Fig. 12c). However, for $x=x_{2}=1.0035181044138$, the corresponding orbit also surrounds the LO but turns upwards, and it has no horseshoe shape (see Fig. 12c). The explanation for this fact is that there must exist an orbit with the initial condition $x$ between $x_{1}$ and $x_{2}$ which belongs to the stable invariant manifold of the LO, and is asymptotic to it (Hénon \& Petit 1986 show the same behaviour in Hill's problem).
Actually, for any two orbits which surround the LO $k$ and $k+1$ times, respectively, there must be another one, between these two orbits, which tends asymptotically to the LO. There exists also a collision orbit with $m_{2}$ for a certain $x \in(1.003518104418,1.0035181044185)$ as Fig. 12c shows. Of course, the Lyapunov orbit around $L_{1}$ also plays a role and several kind of orbits exist when increasing $x$ : periodic horseshoe orbits, symmetric periodic orbits which do not describe a horseshoe shape, collision orbits with the small primary and orbits belonging to and close to the invariant manifolds of the Lyapunov periodic orbits associated with this value of the Jacobian constant.

When $\mathrm{C}$ increases, $C \in\left[C_{3}, C_{1}\right]$ and $C$ is close to $C_{1}$, the $z v c$ is closer to the colinear points $L_{1}$ and $L_{2}$ (see Fig. 1) and the length of the interval $\left[x_{\mathrm{i}}, x_{\mathrm{o}}\right]$, obtained from the intersection of the $z v c$ and the $x$ axis, grows and the number of horseshoe periodic orbit decreases. For instance, for $C=3.00001 \in\left[C_{3}, C_{1}\right]$ very close to $C_{1}$, we have used an increment of variation of the initial condition $x$ of $10^{-9}$ and we have obtained 29 horseshoe 

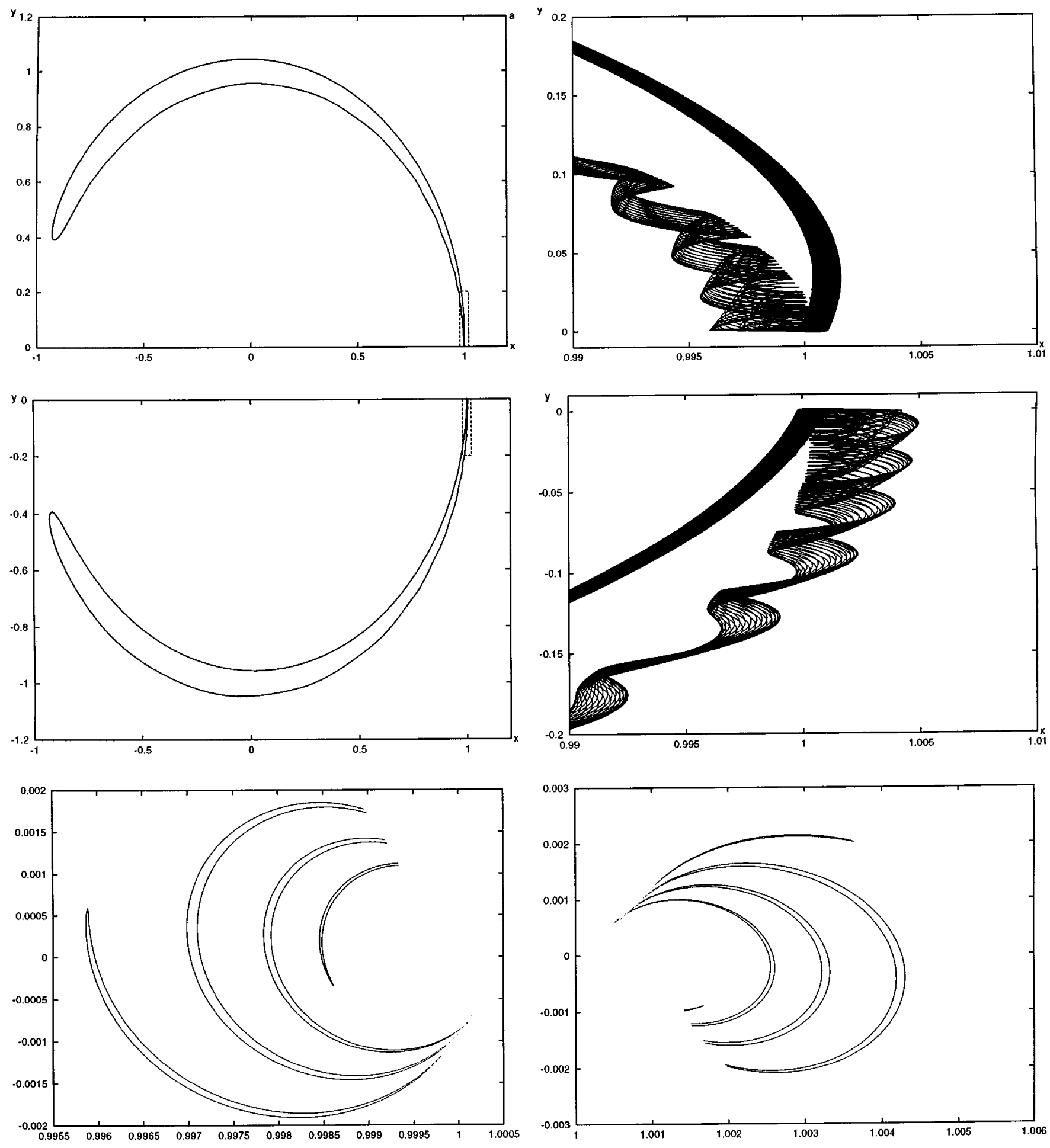

Fig. 16. $\mu=\mu_{\mathrm{SJ}}, C=3.001906457$. a) The flow on $W^{\mathrm{s}, 1}$. b) Detail. c) The flow on $W^{\mathrm{s}, 2}$. d) Detail. e) The curve $\gamma_{\mu, C}^{\mathrm{s}, 1}$ in the $(x, \dot{x})$ plane. f) The curve $\gamma_{\mu, C}^{\mathrm{s}, 2}$ in the $(x, \dot{x})$ plane.

periodic orbits for $x \in[1.0018257,1.00183346]$; actually, as the $z v c$ is closer to the collinear point $L_{1}$ than $L_{2}$, as $x$ increases, there appears, first of all, a symmetric periodic orbit on the left of $m_{2}$, that is $u=0$ and $v<0$ at the second orthogonal crossing (see Fig. 13), then the horseshoe periodic orbits (we note that the return point now becomes a "return path" described close to the Lyapunov orbits of $L_{1}$ and $L_{2}$ ) that coexist with collision orbits and symmetric periodic orbits with the second orthogonal crossing on the right of $m_{2}$ (see Fig. 13), with $u>0$ and 
$v=0$. Again, a more detailed analysis reveals the main role of the Lyapunov periodic orbits of $L_{1}$ and $L_{2}$ and their invariant manifolds; as described above, when $x$ increases, there appear horseshoe-like orbits which tend asimptotically to the Lyapunov orbit of $L_{1}$, first, and to the Lyapunov orbit of $L_{2}$, as $x$ increases further on.

Finally, from some value of $C$ smaller than and very close to $C_{1}$ ( $C$ increasing) horseshoe periodic orbits do not exist any more: for example, for $C=3.000015$ the value of $x_{\mathrm{o}}$ of the $z v c$ is $x_{\mathrm{o}}=1.0022367$ (we note that this value is bigger than any value $x$ of the horseshoe periodic orbits computed for $C=3.00001$ ) and there are no horseshoe periodic orbits. See Sect. 4 for further comments. (ii) For $3 \leq C<C_{3}$, the $z v c$ encloses $L_{4}$ and $L_{5}$ but does not cross the $x$ axis, and for $C<3$ there is no $z v c$ at all. The same pattern is obtained as far as the evolution of the horseshoe periodic orbits is concerned. In both cases, however, besides the Lyapunov orbits (and their stable and unstable manifolds) related to $L_{1}$ and $L_{2}$, there exists also the Lyapunov orbit (and its invariant manifolds) linked to $L_{3}$.

\section{Horseshoe periodic orbits in the RTBP for other values of $\mu$}

A natural question that arises is about the existence of horseshoe periodic orbits in the RTBP for any value of the mass parameter $\mu \in(0,1 / 2]$.

We have applied the same method explained in Sect. 3 for different values of $\mu$. Fixed a value of $\mu$, we obtain the same behaviour, as far as evolution of horseshoe periodic orbits is concerned: on the one hand the number of horseshoe periodic orbits increases as far as $C$ decreases; on the other hand, the usual pattern of the evolution of the horseshoe periodic orbits, for fixed $C$, when increasing the initial condition $x$ is again the one described in Sect. 3.2, that is, they coexist with collision orbits with the small primary as well as with symmetric periodic orbits which do not have a horseshoe shape. In order to show this behaviour, we consider $\mu=0.01$; then $C_{1}=3.164219$ and $C_{3}=3.019898$. We show in Fig. 14 some of the periodic orbits obtained when increasing $x$ for $C=3.0199 \in\left(C_{3}, C_{1}\right)$ - close to $C_{3}$-, and for $C=3.01<C_{3}$. We also remark that, since for $C<3$ there is no $z v c$ and there exist the periodic orbits related to the triangular points $L_{4} \mathrm{nd}$ $L_{5}$, the horseshoe periodic orbits can be quite simple (see Fig. 15a for $\mu=0.01$ ) or rather involved (see Fig. 15b).

In order to figure out the question about the existence of horseshoe periodic orbits, for any value of $\mu>0$, we make two remarks:

(a) first of all, the simple mechanism of generation of horseshoe periodic orbits for $\mu>0$ and very small (that is, the dynamics somewhat inherits the dynamics from $\mu=0)$ is no longer valid for larger values of $\mu$.

(b) Nevertheless, another natural mechanism related to the existence of horseshoe periodic orbits, for any value of $\mu>0$, is due to the (stable and unstable) invariant manifolds of the Lyapunov orbits emanating from the collinear point $L_{3}$ (a detailed study of point $L_{3}$ for $\mu$ small and $C=C_{3}$ is given in Font 1990). For a fixed value of $\mu$ we consider $C<C_{3}$, and we have computed numerically the 2-dimensional invariant manifolds of the Lyapunov periodic orbit that exists for this value of $C$ (see also Llibre et al. 1985 , for a similar work related to $L_{2}$ ). We denoted by $W^{\mathrm{s}, 1}, W^{\mathrm{s}, 2}$ the two stable invariant manifolds and by $W^{\mathrm{u}, 1}$ and $W^{\mathrm{u}, 2}$ the unstable ones; we have followed them until the first intersection with $y=0$ takes place; we denote by $\gamma_{\mu, C}^{\mathrm{s}, \mathrm{i}}$ and $\gamma_{\mu, C}^{\mathrm{u}, \mathrm{i}}$, for $i=1,2$, such curves in the plane $\left(x, x^{\prime}\right)$.

In our computations we have followed the manifolds $W^{\mathrm{s}, 1}$ and $W^{\mathrm{s}, 2}$ (since $W^{\mathrm{u}, 1}$ and $W^{\mathrm{u}, 2}$ are obtained using the symmetry (4)), and the corresponding curves $\gamma_{\mu, C}^{\mathrm{s}, 1}$ and $\gamma_{\mu, C}^{\mathrm{s}, 2}$. Any point obtained from the intersection of $\gamma_{\mu, C}^{\mathrm{s}, i}$ and its symmetrical with respect to the $x$ axis (that is, $\gamma_{\mu, C}^{\mathrm{u}, i}$ ), for $i=1,2$, gives a homoclinic orbit (a symmetrical one if the intersection point has $x^{\prime}=0$ ) which tends in forward and backward time to the Lyapunov orbit; and therefore a family of periodic orbits tending to this homoclinic orbit. This explains the existence of horseshoe periodic orbits when varying the initial value $x$ for fixed $C<C_{3}$. Of course, when $C \geq C_{3}$, although there are neither the Lyapunov orbits (related to $L_{3}$ ) nor the invariant manifolds, some of the horseshoe periodic orbits remain but their number decreases for increasing $C$.

Our results reveal three kinds of phenomena according to the value of $\mu$ and $C \in\left[3, C_{3}\right)$ :

(i) For $0<\mu \leq 0.004$ and $3 \leq C<C_{3}$, the flow on the invariant manifolds has a horseshoe shape and the curves $\gamma_{\mu, C}$ are defined for $x>0$ and give rise to homoclinic orbits. We show this behaviour for $\mu=0.000953875$ (Sun-Jupiter mass parameter) and $C=3.001906457$ (see Fig. 16); in particular the curves $\gamma_{\mu, C}^{\mathrm{s}, 1}$ (Figs. 16e,f) show the existence of homoclinic orbits not detected in Henrard's paper (1983).

(ii) As far as $\mu$ increases, the domain of attraction of the small primary $m_{2}$ is more powerful, and the flow on the invariant manifolds of the Lyapunov family of periodic orbits (emanating from $L_{3}$ ) may get very close to and even collide with it, although some orbits on the invariant manifolds keep a horseshoe shape. This is the case for $\mu \in$ $[0.0045,0.099]$. We show, for example, in Fig. 17 the flow on $W^{\mathrm{s}, 1}$, for $\mu=0.01$ and $C=3.01<C_{3}$; although there are some orbits belonging to $W^{\mathrm{s}, 1}$ which go to collision with $m_{2}$, there remain other that have a horseshoe shape and also homoclinic orbits obtained from the curves $\gamma_{\mu, C}^{\mathrm{s}, 1}$ at the first intersection with $y=0, x>0$ (see Fig. 17b) or even at the second one with $y=0, x>0$ (see Fig. 17c). Such homoclinic orbits allow the existence of horseshoe periodic orbits with an initial $x$ value far from the $z v c$; this agrees with the orbits displayed in Fig. 14b, in particular for the horseshoe periodic orbits with initial conditions $x=2.009666525311$ and $x=2.05523296943$.

(iii) Finally for $\mu \geq 0.1$ and $3 \leq C<C_{1}$, the invariant manifolds of the Lyapunov orbits emanating from $L_{3}$ may give a hint on the existence of horseshoe orbits. For 

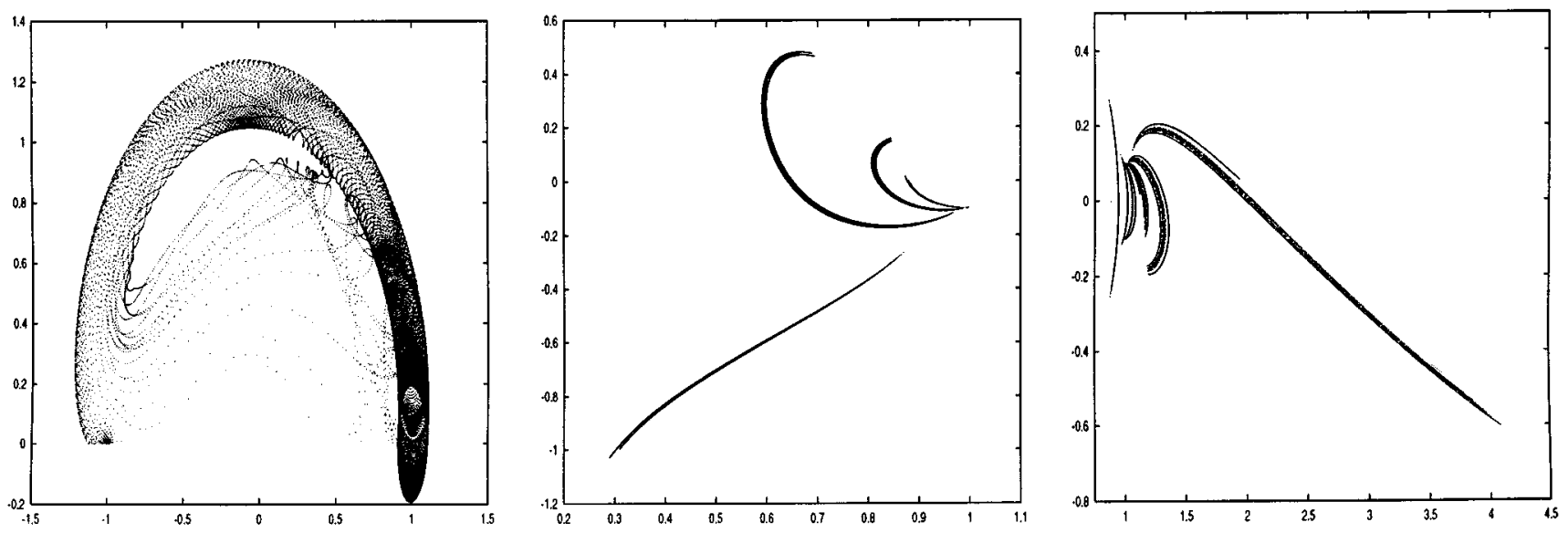

Fig. 17. $\mu=0.01, C=3.01$. a) The flow on $W^{\mathrm{s}, 1}$ in the $(x, y)$ plane. b) The curve $\gamma_{\mu, C}^{\mathrm{s}, 1}$ in the $(x, \dot{x})$ plane. c) The curve, in the $(x, \dot{x})$ plane, obtained from the second intersection between $W^{\mathrm{s}, 1}$ and $y=0, x>0$.
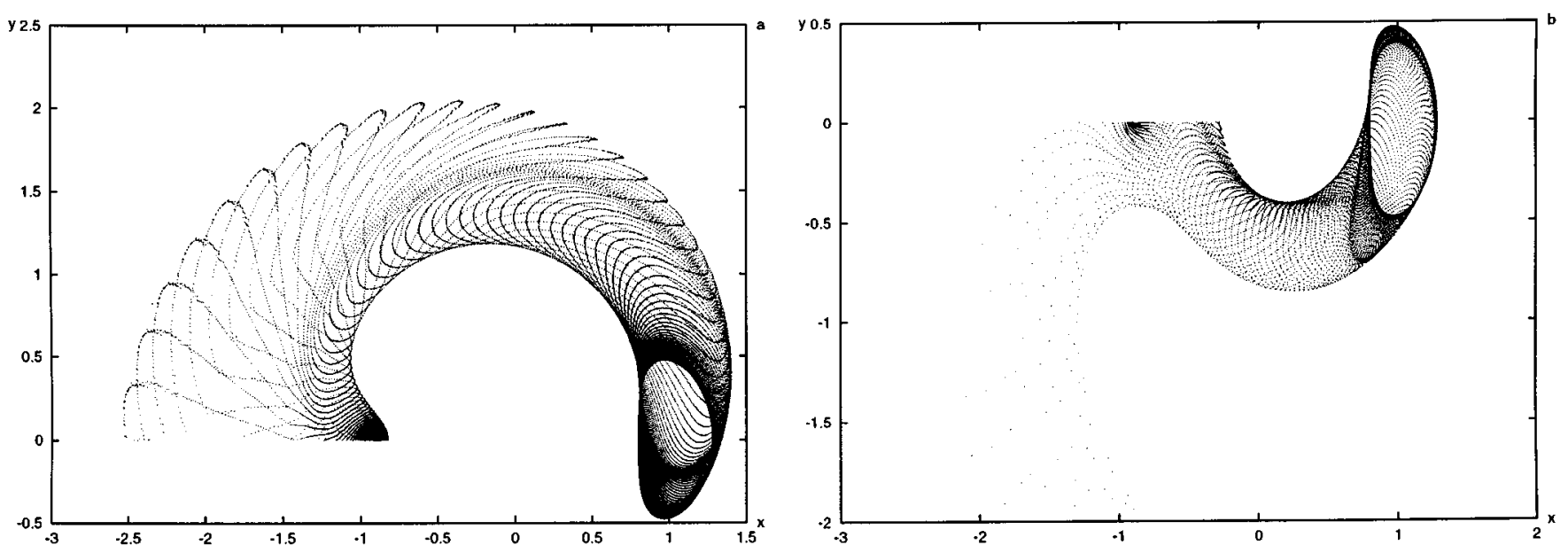

Fig. 18. $\mu=0.1, C=3.1<C_{3}$. a) The flow on $W^{\mathrm{s}, 1}$. b) The flow on $W^{\mathrm{s}, 2}$.

example, for $\mu=0.1$ and $C_{3} \leq C \leq C_{1}$, there are no horseshoe periodic orbits and we show in Fig. 18 how the flow on the invariant manifolds of the corresponding Lyapunov orbit, for $C=3.1<C_{3}$, does not describe a horseshoe shape anymore.

\section{Conclusions}

According to the two purposes of this paper (see Sect. 1), we can conclude the following:

(i) The horseshoe shaped motion of the Saturn coorbital satellites Janus and Epimetheus is already well reflected in the simple model of the circular restricted threebody problem, since we have been able to compute stable horseshoe periodic orbits that are quantitatively and qualitatively very close to their motion.

Of course, a better model to describe the coorbitals' motion should include other effects: the eccentric orbits and the masses of both satellites, their inclination and the oblateness of Saturn as the main ones. However this will be the subject of research in the near future. (ii) The natural mechanism to generate horseshoe periodic orbits for very small $\mu$ remains in the dynamics for $\mu=0$, and it also provides information about their existence and location.

(iii) The invariant manifolds of the Lyapunov family of periodic orbits (related to $L_{1}, L_{2}$ and $L_{3}$, and specially to $L_{3}$ ) play a key role in the existence of horseshoe periodic orbits in the RTBP for any value of $\mu>0$ and $3 \leq C<C_{1}$. They have many homoclinic orbits which in turn cause a rich structure of families of periodic orbits. In particular, such families allow the existence of the horseshoe periodic orbits described in this paper.

Acknowledgements. The first author is partially supported by DGES grant number PB96-1153 and by CIRIT grant number 1999SGR-00349.

The second author acknowledges the Departament de Matemàtiques (Universitat Autònoma de Barcelona) and the Centre de Recerca Matemàtica where this work has been carried out, and is partially supported by the Catalan grant CIRIT number 2000SGR-00027 and by the Spanish grant number BFM2000-0623. 


\section{References}

Aksnes, K. 1985, in Stability of the Solar System and its Minor Natural and Artificial Bodies, ed. V. Szebehely (Reidel Publ. Co.), 3

Bray, T. A., \& Goudas, C. L. 1967, Adv. Astron. Astrophys., 5,71

Cors, J. M., \& Hall, D., in preparation

Dermott, S. F., Murray, C. D., \& Sinclair, A. T. 1980, Nature, 284,309

Dermott, S. F., \& Murray, C. D. 1981a, Icarus, 48, 1

Dermott, S. F., \& Murray, C. D. 1981b, Icarus, 48, 12

Font, J. 1990, Ph.D. Thesis, Barcelona University

Garfinkel, B. 1977, AJ, 82, 368

Garfinkel, B. 1978, Cel. Mech., 18, 259

Garfinkel, B. 1980, Cel. Mech., 22, 267

Hénon, M., \& Petit, J. M. 1986, Cel. Mech., 38, 67

Henrard, J. 1983, Cel. Mech., 31, 115
Llibre, J., Martinez, R., \& Simo, C. 1985, J. Diff. Eqs., 58, 104 Murray, C. D., \& Dermott, S. F. 1999, Solar system dynamics (Cambridge Press)

Nicholson, P. D., et al. 1992, Icarus, 100, 464

Petit, J. M., \& Hénon, M. 1986, Icarus, 66, 536

Rabe, E. 1961, AJ, 66, 500

Spirig, F., \& Waldvogel, J. 1985, in Stability of the Solar System and its minor and Artificial Bodies, ed. V. Szebehely (Reidel), 53

Szebehely, V. 1967, Theory of orbits (Academic Press)

Taylor, D. B. 1981, A\&A, 103, 288

Waldvogel, J., \& Spirig, F. 1988, in Long-Term Dynamical Behaviour of Natural and Artificial N-Body Systems, ed. A. E. Roy (Kluwer Acad. Pub.), 223

Yoder, C. F., Colombo, G., Synnot, S. P., \& Yoder, K. A. 1983, Icarus, 53, 431

Yoder, C. F., Synnot, S. P., \& Salo, H. 1989, AJ, 98, 1875 